\title{
EFTEM and EELS Studies of the Interface Structure of Ni/MgO
}

\author{
K. Sun,* and L. M. Wang** \\ * Electron Microbeam Analysis Laboratory, University of Michigan, Ann Arbor, MI 48109 \\ ** Department of Nuclear Engineering and Radiological Sciences, University of Michigan, Ann \\ Arbor, MI 48109
}

Ni thin films growing epitaxially on some substrates, for example, on single crystal $\mathrm{MgO}$, are of great interests recently due to their special magnetic properties that may lead to some new applications. Although $\mathrm{MgO}$ substrate and $\mathrm{Ni}$ have a large lattice mismatch of $\sim 16.4 \%$, cube on cube (CC) epitaxial growth has been successfully conducted [1]. Theoretical investigations predict that $\mathrm{Ni}$ interacts strongly with $\mathrm{MgO}$ [2], i.e., $\mathrm{Ni}$ is easy to be oxidized. Moreover based on the measurement of the magnetic property, polarized neutron reflectivity and high-resolution X-ray diffraction studies, it was speculated that there probably forms an ordered $\mathrm{NiO}$ thin layer $(\sim 5 \mathrm{~nm})$ at the interface [1]. Nevertheless, it is still not clear whether or not such a thin NiO layer is formed at the $\mathrm{Ni} / \mathrm{MgO}$ interfaces. A detailed study of the local electronic structure of the interface is needed.

The growth of a thin (about $50 \mathrm{~nm}$ ) Ni film on $\mathrm{MgO}(001)$ substrate was conducted in a MBE VG $80 \mathrm{M}$ system. The sample was annealed at $300^{\circ} \mathrm{C}$ after growth. More detailed descriptions can be found in reference [1]. A JEOL-2010F STEM/TEM was used for the study that can be performed in both TEM and STEM modes at $200 \mathrm{kV}$. A Gatan Image Filter (GIF) system is attached to this microscope that can be used for electron energy loss spectroscopy (EELS) [3] and energy filtered transmission electron microscope (EFTEM) [4]. EFTEM images were recorded at an 8k magnification using a CCD camera at $2 \times$ binning $(512 \times 512$ pixels). The lens conditions were set to define probe sizes of $0.2 \mathrm{~nm}$ for both high-angle annual dark-field (HAADF) imaging and EELS.

Figure 1 shows EFTEM elemental mapping results. From Fig.1 (a) it can be clearly seen that an interfacial layer ( $\sim 5 \mathrm{~nm}$ thick) is formed at the interface of $\mathrm{Ni} / \mathrm{MgO}$ that has distinct interfaces with both the substrate and the expitaxial thin film. To identify the structure of such a thin layer, elemental mapping was performed for both $\mathrm{Ni}$ and $\mathrm{O}$ elements across the interface of $\mathrm{Ni} / \mathrm{MgO}$. The Ni- (Fig. 1 (b)) and O- (Fig. 1 (c)) maps show that distributions of $\mathrm{Ni}$ and $\mathrm{O}$ elements all cover such a thin layer. This suggests that the thin layer consists of both $\mathrm{Ni}$ and $\mathrm{O}$ elements that can also be clearly seen from the RBG map reconstructed from the two elemental maps (Fig.1 (d)). Although we have not succeeded in acquiring a high quality $\mathrm{Mg}$ map from the same area due to that $\mathrm{Mg}$ has a relatively high energy core-loss peak ( $K$ edge), conclusion can still be made that the formed thin layer at the interface is $\mathrm{NiO}$ layer. Diffusion of $\mathrm{Ni}$ atoms down to several nanometers into the $\mathrm{MgO}$ substrate is not possible under the present experimental conditions.

Figure 2 (a) clearly shows the $\mathrm{CC}$ epitaxial growth of a high quality $\mathrm{Ni}$ thin film on the $\mathrm{MgO}$ substrate. A thin layer with lattice matching well with the $\mathrm{MgO}$ substrate is clearly seen. Due to that both $\mathrm{NiO}$ and $\mathrm{MgO}$ have fcc $(\mathrm{Fm} 3 \mathrm{~m})$ structures with similar lattice parameters $(0.4177$ and 0.4213 $\mathrm{nm}$, respectively), it is not possible to resolve them from HREM images. To obtain electronic structure information from the interface, EELS profile (O: $K$ edge and Ni: $L_{23}$ edges were used) was performed in STEM mode that are displayed in Fig. 2 (b) and (c). Because the white-line intensity ratio $\left(L_{3} / L_{2}\right)$ for the Ni oxide is a little bit larger than that for the Ni metal [5], we can extract the 
valance state information of $\mathrm{Ni}$ by calculating the white-line intensity ratios of the spectra.

Measurement of the $L_{3} / L_{2}$ intensity ratios in second derivative mode gives the intensity ratios of 3.33.5 (for the spectra acquired from position a) and 3.6-4.0 (for the spectra acquired from position $\mathbf{b}$ ), respectively. This further confirms that the interfacial thin layer is oxidized, i.e., a thin NiO layer is formed. Further studies involving using high-energy resolution atomic resolved EELS and electron spectroscopic imaging (ESI) techniques are ongoing. More information about the bonding in the $\mathrm{Ni} / \mathrm{NiO}$ and the $\mathrm{NiO} / \mathrm{MgO}$ interfaces as well as the $\mathrm{MgO}$ side structure will be provided.

\section{References}

[1] Z. Zhang, et al., J. Vac. Sci. Technol. A22 (2004) 1868.

[2] G. Pacchioni, et al., J. Chem. Phys. 104 (1996) 7329.

[3] R. F. Egerton, Electron Energy Loss Spectroscopy in the Electron Microscope, 2nd, Plenum:

New York, 1996.

[4] L. Reimer, Energy-Filtering Transmission Electron Microscopy, Vol. 71, Springer Series in Optical Sciences, Springer-Verlag, Berlin, 1995.

[5] R. D. Leapman, et al., Phys. Rev. B26 (1982) 614.

[6] The JEOL-2010F STEM/TEM was funded by NSF through the Grant DMR-9871177. Z. Zhang of the University of Toledo supplied the $\mathrm{Ni} / \mathrm{MgO}$ sample.
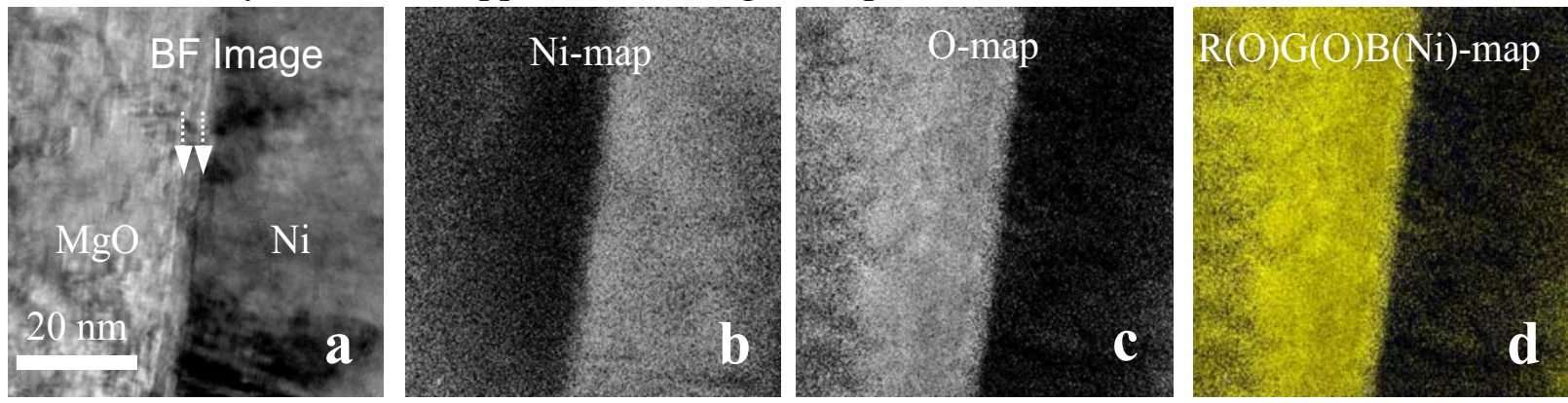

FIG. 1. (a) - (d) display BF, Ni-map, O-map and the generated RGB-map, respectively, obtained from the $\mathrm{Ni} / \mathrm{MgO}$ interface.
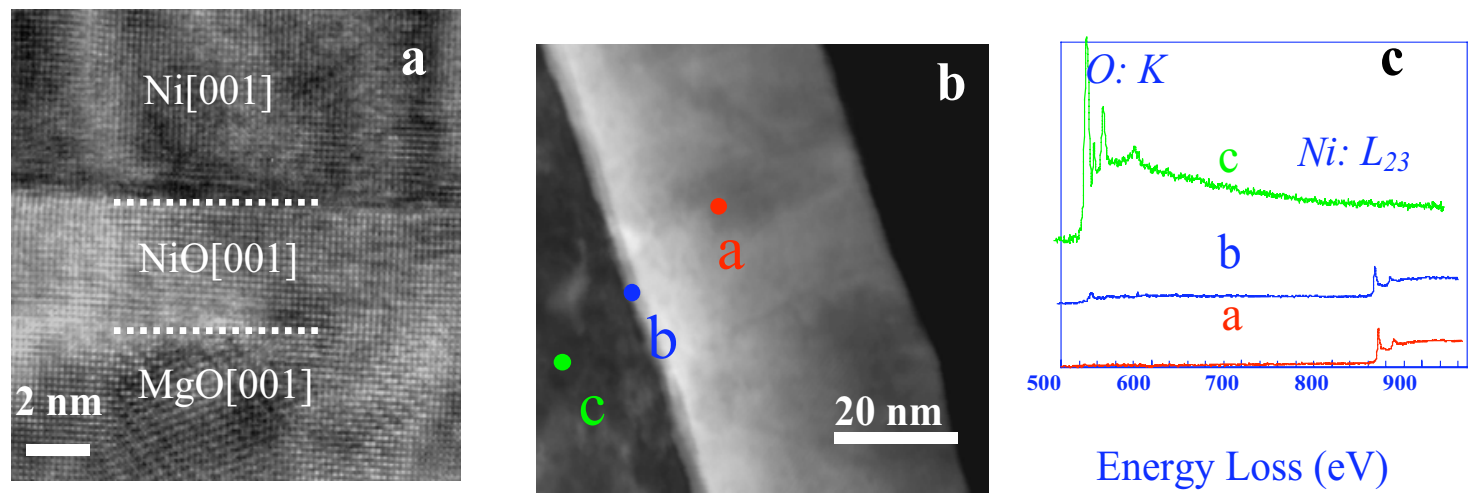

FIG. 2. (a) HREM image showing the interface structure of the $\mathrm{Ni} / \mathrm{MgO}$; (b) HAADF image with three positions marked from which corresponding EEL spectra were acquired shown in (c). The EEL spectra were background removed by fitting the background to a power law of the form $A e^{-r}$ and then were normalized to the energy range of $900-950 \mathrm{eV}$. They were vertically shifted for clarity. 